\title{
Association of Ambulatory Blood Pressure with All-Cause Mortality and Cardiovascular Outcomes in Peritoneal Dialysis Patients
}

\author{
Shuqi Dai Yun Chen Da Shang Xiaolin Ge Qionghong Xie Chuan- \\ Ming Hao Tongying Zhu
}

Department of Nephrology, Huashan Hospital, Fudan University, Shanghai, China

\section{Keywords}

Peritoneal dialysis - Ambulatory blood pressure monitoring - Mortality · Pulse pressure ·

Calcification

\begin{abstract}
Background: Ambulatory blood pressure monitoring is the gold standard for the diagnosis of hypertension, but its effects on all-cause mortality and cardiovascular outcomes in peritoneal dialysis (PD) patients remain uncertain. We aimed to investigate the association between ambulatory blood pressure and clinical outcomes in PD patients. Methods: A prospective, observational cohort study was conducted in PD patients enrolled from March 2001 to July 2018 and followed until October 2019. Blood pressure was evaluated using 24-h ambulatory blood pressure monitoring. The endpoints included all-cause mortality, cardiovascular mortality, and cardiovascular events. Multivariable Cox regression was used to identify the associations between ambulatory blood pressure and endpoints. Subsequently, multivariable logistic regression was conducted to identify factors associated with elevated pulse pressure (PP). Results: A total of 260 PD patients (154 men, 59.2\%) were recruited. The median follow-up duration was 40.7 months. Our studies revealed that PP was an independent predictor of all-cause mortality (hazard ratio $[\mathrm{HR}], 1.018 ; 95 \% \mathrm{Cl}, 1.001-1.034 ; p=0.032)$, cardiovascular mortality $(\mathrm{HR}, 1.039 ; 95 \%$ $\mathrm{Cl}, 1.017-1.061 ; p<0.001)$, and cardiovascular events ( $\mathrm{HR}, 1.028 ; 95 \% \mathrm{Cl}, 1.011-1.046 ; p=0.001)$. Systolic blood pressure was an independent predictor of cardiovascular mortality (HR, 1.023;
\end{abstract}

Shuqi Dai and Yun Chen contributed equally to this work. 
$95 \% \mathrm{Cl}, 1.007-1.040 ; p=0.005)$ and cardiovascular events $(\mathrm{HR}, 1.018 ; 95 \% \mathrm{Cl}, 1.006-1.030 ; p=$ 0.003). Vascular calcification was significantly associated with elevated PP $(O R, 3.069 ; 95 \% \mathrm{Cl}$, $1.632-5.772 ; p=0.001)$. Conclusion: $24-\mathrm{h}$ ambulatory PP was the most significant predictor of all blood pressure indicators for clinical outcomes in PD patients.

(C) 2020 The Author(s).

Published by S. Karger AG, Basel

\section{Introduction}

Cardiovascular death is the leading cause of mortality in patients on peritoneal dialysis (PD) [1]. Hypertension is a general and controllable cardiovascular risk factor and is highly prevalent in PD patients [2,3]. Compared to the general population, blood pressure control is more challenging in PD patients due to complex pathophysiology such as fluid overload [4]. Therefore, clarifying the relationship between blood pressure and prognosis in PD patients is particularly important for blood pressure management.

In previous studies, associations between blood pressure and outcomes in the dialysis population remain uncertain, probably because of short duration of follow-up or small sample size $[5,6]$. It has been demonstrated that ambulatory blood pressure monitoring is the gold standard for the diagnosis of hypertension [7, 8]. In the general population, higher 24-h blood pressure value was significantly associated with greater risks of death and cardiovascular outcomes [9]. In the hemodialysis population, several studies reported a U-shaped relationship between systolic blood pressure (SBP) or pulse pressure (PP) and mortality $[10,11]$. Yet, little prospective evidence on the utility of ambulatory blood pressure monitoring for risk prediction exists in PD patients. Thus, the present study aimed to examine the associations between 24 -h blood pressure and all-cause mortality, cardiovascular mortality, and cardiovascular events of PD patients and to explain the underlying cause of the association.

\section{Materials and Methods}

\section{Population and Study Design}

Adult PD patients from Huashan Hospital, Fudan University in China were recruited in this prospective, observational, longitudinal cohort study. Patients were enrolled from March 2001 to July 2018 and followed until death, changes in treatment modality, renal transplantation, or October 2019. Patients were excluded if they had an ongoing infection or were clinically unstable, with a life expectancy of fewer than 6 months. All of the patients received regular PD for $>3$ months and provided written informed consent. The study protocol was approved by the Ethics Committee of Huashan Hospital affiliated to Fudan University.

\section{Outcome Measurements}

All-cause mortality was the primary endpoint of the study. The secondary endpoints included cardiovascular mortality and cardiovascular events. Cardiovascular events included coronary artery events (e.g., new ST-elevation myocardial infarction, non-ST-elevation myocardial infarction, unstable angina, and stable angina), heart failure, cerebrovascular diseases (ischemic and hemorrhagic strokes), and peripheral artery disease (e.g., arterial embolization and diabetic foot). Cardiovascular mortality was defined as death caused by cardiovascular events. Patients who received renal transplantation, switched to hemodialysis (HD), transferred, or died were censored for survival analysis.

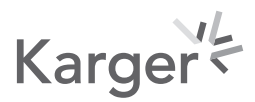




\section{Blood Pressure Measurements}

Blood pressure was assessed for $24 \mathrm{~h}$ using SCHILLER BR-102 plus (Schiller AG, Baar, Switzerland), which is validated according to standard protocols $[12,13]$. Daytime $(07: 00-$ 23:00) blood pressure was measured every 10-20 min and nighttime (23:00-07:00) blood pressure was measured every 30-60 min. Ambulatory recordings had to contain over $80 \%$ of valid readings for further analysis. Recorded parameters include 24-h average SBP, diastolic blood pressure (DBP), and PP (PP = SBP - DBP).

\section{Clinical and Laboratory Data}

Clinical characteristics including age, sex, BMI, history of diabetes, medication status, and smoking status were obtained at the time of enrollment. PD adequacy parameters including total $\mathrm{Kt} / \mathrm{V}$, residual $\mathrm{Kt} / \mathrm{V}$, total clearance of creatinine, and residual clearance of creatinine were evaluated using Baxter PD Adequest 2.0 software (Baxter Healthcare Corporation, Deerfield, IL, USA) at baseline. Vascular calcification was evaluated by coronary artery calcification using multi-slice spiral CT. When the coronary calcification score was $>0$, the presence of calcification was considered.

Laboratory data including hemoglobin, serum albumin, calcium, phosphorus, potassium, intact parathyroid hormone, glycated hemoglobin (HbA1c), serum insulin, cholesterol, triglyceride, high-density lipoprotein, low-density lipoprotein (LDL), N-terminal pro-brain natriuretic peptide (NT-proBNP), and high-sensitivity C-reactive protein were determined using standard laboratory methods. Homeostasis model of assessment-insulin resistance (HOMA-IR) was used to evaluate insulin resistance using the validated equation: fasting serum insulin $(\mathrm{mU} / \mathrm{L}) \times$ fasting serum glucose $(\mathrm{mmol} / \mathrm{L}) / 22.5$.

\section{Statistical Analysis}

SPSS (version 26.0) and R (version 3.6.2) were used to perform the statistical analysis. Continuous variables were presented as mean \pm standard deviation or as median (interquartile range) according to the normality test results, with categorical variables as percentages. Comparisons between the groups were carried out using a $t$ test or Mann-Whitney $\mathrm{U}$ test for continuous variables and Pearson's $\chi^{2}$ test or Fisher's exact test for categorical variables. Univariable Cox regression was used to identify the potential risk factors for endpoints (allcause mortality, cardiovascular mortality, and cardiovascular events) in PD patients, and multivariable Cox regression was used to identify independent predictors. Variables previously demonstrated to be significant in univariable Cox analysis or thought to be clinically important were included in the multivariable Cox regression model. The hazard ratios (HRs) for the endpoints were expressed per $1 \mathrm{~mm} \mathrm{Hg}$ increase in blood pressure. For the generation of the Kaplan-Meier (KM) survival curves, participants were stratified into 2 groups based on the median PP level. Nonlinear associations between blood pressure and outcomes were determined using restricted cubic spline methods. Furthermore, multivariable logistic regression was conducted to verify the risk factors associated with elevated PP. All of the statistical tests were 2-sided, and values were considered statistically significant at the $p$ value $<0.05$.

\section{Results}

\section{Characteristics of Study Participants}

Our study population included 260 patients (154 men, 59.2\%) with a median age of 63.61 $(46.64,73.2)$ years. The patients were divided into 2 groups according to the median PP level (ranging from 25 to 100): low (PP $\leq 49 \mathrm{~mm} \mathrm{Hg}, n=132$ ) and high (PP $>49 \mathrm{~mm} \mathrm{Hg}, n=128$ ). Baseline characteristics of all patients and those with low PP and high PP are shown in Table 1.

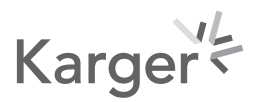


Table 1. Baseline characteristics of the study participants

\begin{tabular}{|c|c|c|c|c|}
\hline & \multirow[t]{2}{*}{ Total $(n=260)$} & \multicolumn{2}{|c|}{ 24-h pulse blood pressure } & \multirow[t]{2}{*}{$p$ value } \\
\hline & & $\leq 49 \mathrm{~mm} \mathrm{Hg}(n=132)$ & $>49 \mathrm{~mm} \mathrm{Hg}(n=128)$ & \\
\hline \multicolumn{5}{|l|}{ Clinical characteristics } \\
\hline Age, years & $63.61(46.64,73.2)$ & $53.85(40.83,68.38)$ & $70.16(59.74,78.61)$ & $<0.001$ \\
\hline BMI, $\mathrm{kg} / \mathrm{m}^{2}$ & $22.99 \pm 3.59$ & $22.60 \pm 3.76$ & $23.39 \pm 3.38$ & 0.078 \\
\hline Male, $n(\%)$ & $154(59.2)$ & $81(61.4)$ & $73(57.0)$ & 0.477 \\
\hline History of diabetes, $n(\%)$ & $69(26.5)$ & $12(9.1)$ & $57(44.5)$ & $<0.001$ \\
\hline History of smoking, $n(\%)$ & $46(17.7)$ & $24(18.2)$ & $22(17.2)$ & 0.834 \\
\hline Use of statins, $n(\%)$ & $99(38.1)$ & $41(31.1)$ & $58(45.3)$ & 0.018 \\
\hline Use of anticoagulation drug, $n(\%)$ & $55(21.2)$ & $22(16.7)$ & $33(25.8)$ & 0.072 \\
\hline Use of calcitriol, $n(\%)$ & $120(46.2)$ & $60(46.9)$ & $60(45.5)$ & 0.818 \\
\hline Use of calcium acetate, $n(\%)$ & $97(37.3)$ & $47(35.6)$ & $50(39.1)$ & 0.565 \\
\hline Use of antihypertensive drug, $n(\%)$ & $245(94.2)$ & $120(90.9)$ & $125(97.7)$ & 0.020 \\
\hline Residual renal Kt/V & $0.57(0.18,0.96)$ & $0.62(0.19,1.02)$ & $0.51(0.18,0.91)$ & 0.286 \\
\hline Total Kt/V & $1.93(1.69,2.29)$ & $1.95(1.72,2.30)$ & $1.92(1.67,2.28)$ & 0.747 \\
\hline Residual renal Ccr, L/week & $29.86(12.67,49.02)$ & $34.78(14.79,50.21)$ & $26.27(11.39,47.57)$ & 0.245 \\
\hline Total Ccr, L/week & $67.64(53.34,85.47)$ & $69.34(55.46,86.72)$ & $61.94(51.37,81.77)$ & 0.096 \\
\hline Calcification, $n(\%)$ & $134(51.5)$ & $41(31.1)$ & $93(72.7)$ & $<0.001$ \\
\hline PD duration, months & $7.73(3.0,33.07)$ & $9.28(3,26.43)$ & $7.67(3,37.52)$ & 0.700 \\
\hline All-cause mortality, $n(\%)$ & $64(24.6)$ & $17(12.9)$ & $47(36.7)$ & $<0.001$ \\
\hline Cardiovascular mortality, $n(\%)$ & $33(12.7)$ & $6(4.5)$ & $27(21.1)$ & $<0.001$ \\
\hline Cardiovascular events, $n(\%)$ & 57 (21.9) & $13(9.8)$ & $44(34.4)$ & $<0.001$ \\
\hline \multicolumn{5}{|l|}{ Laboratory data } \\
\hline Hemoglobin, g/L & $109.12 \pm 19.22$ & $111.50 \pm 17.74$ & $106.66 \pm 20.41$ & 0.042 \\
\hline Serum albumin, g/L & $36(33,40)$ & $37.5(34,41)$ & $34.5(32,38)$ & $<0.001$ \\
\hline Calcium, mmol/L & $2.22 \pm 0.21$ & $2.23 \pm 0.21$ & $2.20 \pm 0.20$ & 0.213 \\
\hline Phosphorus, mmol/L & $1.44(1.21,1.71)$ & $1.50(1.24,1.79)$ & $1.41(1.14,1.67)$ & 0.040 \\
\hline Potassium, mmol/L & $4.2(3.8,4.6)$ & $4.2(3.7,4.6)$ & $4.2(3.8,4.6)$ & 0.890 \\
\hline iPTH, pg/mL & $292.3(159.3,428.6)$ & $293.3(162.5,449.3)$ & $279(156.3,390)$ & 0.190 \\
\hline $\mathrm{HbA} 1 \mathrm{c}, \%$ & $5.5(5.2,6.1)$ & $5.3(5.1,5.7)$ & $5.8(5.3,6.7)$ & $<0.001$ \\
\hline Serum insulin, mU/L & $10.0(6.8,15.78)$ & $9.4(6.6,14.23)$ & $10.7(7.7,17.15)$ & 0.017 \\
\hline HOMA-IR & $2.44(1.53,4.04)$ & $2.13(1.42,3.36)$ & $2.99(1.78,5.80)$ & $<0.001$ \\
\hline Cholesterol, mmol/L & $4.31(3.58,5.1)$ & $4.25(3.41,4.9)$ & $4.56(3.9,5.2)$ & 0.017 \\
\hline Triglyceride, mmol/L & $1.69(1.14,2.36)$ & $1.67(1.27,2.30)$ & $1.70(1.04,2.43)$ & 0.808 \\
\hline $\mathrm{HDL}, \mathrm{mmol} / \mathrm{L}$ & $0.95(0.78,1.20)$ & $0.94(0.76,1.16)$ & $0.97(0.78,1.23)$ & 0.245 \\
\hline $\mathrm{LDL}, \mathrm{mmol} / \mathrm{L}$ & $2.26(1.74,2.79)$ & $2.16(1.66,2.62)$ & $2.34(1.83,2.95)$ & 0.030 \\
\hline NT-proBNP (per 1,000 pg/mL) & $1.45(0.60,3.78)$ & $0.96(0.41,2.09)$ & $2.22(0.88,5.69)$ & $<0.001$ \\
\hline hsCRP, mg/L & $1.03(0.36,3.22)$ & $1.00(0.26,3.00)$ & $1.08(0.43,3.29)$ & 0.272 \\
\hline
\end{tabular}

Values denote the mean \pm SD or median (interquartile range) unless specified otherwise. Kt/V, urea clearance; Ccr, creatinine clearance; PD, peritoneal dialysis; iPTH, intact parathyroid hormone; HbA1c, glycated hemoglobin; HOMA-IR, homeostasis model of assessment-insulin resistance; HDL, high-density lipoprotein; LDL, low-density lipoprotein; NT-proBNP, N-terminal pro brain natriuretic peptide; hsCRP, high-sensitivity C-reactive protein.

Compared to the low PP group, patients with a higher PP were older and more likely to have vascular calcification and to have a history of diabetes. They had higher HbA1c, serum insulin, HOMA-IR, cholesterol, LDL, and NT-proBNP but lower albumin and phosphorus. Meanwhile, more people in high PP group were on statins and antihypertensive drugs.

The median follow-up duration was 40.7 (26.1, 67.8) months. During the follow-up period, 57 patients experienced cardiovascular events and 64 patients died, with 33 deaths attributable to cardiovascular diseases. More all-cause mortality, cardiovascular mortality, and cardiovascular events occurred in the high PP group. 


\section{Kidney and Blood Pressure Research}

Dai et al.: Ambulatory Blood Pressure in Peritoneal Dialysis

Table 2. Adjusted proportional hazard ratios for mortality and cardiovascular events for SBP, DBP, and PP per mm Hg increase

\begin{tabular}{|c|c|c|c|c|c|c|}
\hline \multirow[t]{2}{*}{ Predictor } & \multicolumn{2}{|l|}{ All-cause mortality } & \multicolumn{2}{|c|}{ Cardiovascular mortality } & \multicolumn{2}{|l|}{ Cardiovascular events } \\
\hline & $\mathrm{HR}(95 \% \mathrm{CI})$ & $p$ value & $\operatorname{HR}(95 \% \mathrm{CI})$ & $p$ value & $\mathrm{HR}(95 \% \mathrm{CI})$ & $p$ value \\
\hline \multicolumn{7}{|l|}{ 24-h SBP } \\
\hline Model 1 & $1.012(1.000-1.024)$ & 0.044 & $1.029(1.013-1.045)$ & $<0.001$ & $1.021(1.009-1.033)$ & $<0.001$ \\
\hline Model 2 & $1.014(1.002-1.026)$ & 0.019 & 1.030 (1.014-1.047) & $<0.001$ & $1.021(1.009-1.033)$ & 0.001 \\
\hline Model 3 & $1.014(1.002-1.026)$ & 0.019 & $1.030(1.014-1.047)$ & $<0.001$ & $1.021(1.009-1.033)$ & 0.001 \\
\hline Model 4 & $1.014(1.002-1.026)$ & 0.020 & $1.030(1.014-1.047)$ & $<0.001$ & $1.021(1.009-1.033)$ & 0.001 \\
\hline Model 5 & - & 0.105 & $1.023(1.007-1.040)$ & 0.005 & $1.018(1.006-1.030)$ & 0.003 \\
\hline \multicolumn{7}{|l|}{ 24-h $D B P$} \\
\hline Model 1 & - & 0.745 & - & 0.085 & - & 0.076 \\
\hline Model 2 & - & 0.443 & - & 0.089 & - & 0.079 \\
\hline Model 3 & - & 0.443 & - & 0.089 & - & 0.079 \\
\hline Model 4 & - & 0.490 & - & 0.089 & - & 0.079 \\
\hline Model 5 & - & 0.727 & - & 0.900 & - & 0.164 \\
\hline \multicolumn{7}{|l|}{ 24-h PP } \\
\hline Model 1 & $1.020(1.005-1.036)$ & 0.010 & 1.047 (1.027-1.067) & $<0.001$ & $1.032(1.015-1.050)$ & $<0.001$ \\
\hline Model 2 & $1.022(1.006-1.039)$ & 0.006 & $1.043(1.023-1.064)$ & $<0.001$ & $1.032(1.014-1.049)$ & $<0.001$ \\
\hline Model 3 & $1.022(1.006-1.039)$ & 0.006 & $1.043(1.023-1.064)$ & $<0.001$ & $1.032(1.014-1.049)$ & $<0.001$ \\
\hline Model 4 & $1.023(1.007-1.040)$ & 0.006 & $1.043(1.023-1.064)$ & $<0.001$ & $1.032(1.014-1.049)$ & $<0.001$ \\
\hline Model 5 & $1.018(1.001-1.034)$ & 0.032 & $1.039(1.017-1.061)$ & $<0.001$ & $1.028(1.011-1.046)$ & 0.001 \\
\hline
\end{tabular}

The multivariable Cox regression analysis and sequentially adjusted models for the covariates were used to calculate the HRs for the endpoints. Model 1, adjusted for age and sex. Model 2, adjusted for age, sex, BMI, and duration of PD. Model 3, adjusted for age, sex, BMI, duration of PD, HbA1c, and HOMA-IR. Model 4, adjusted for age, sex, BMI, duration of PD, HbA1c, HOMA-IR, and albumin. Model 5, adjusted for age, sex, BMI, duration of PD, HbA1c, HOMA-IR, albumin, and NT-proBNP. SBP, systolic blood pressure; DBP, diastolic blood pressure; PP, pulse pressure; HR, hazard ratio; PD, peritoneal dialysis; HbA1c, glycated hemoglobin; HOMA-IR, homeostasis model of assessment-insulin resistance; NT-proBNP, N-terminal pro brain natriuretic peptide.

Association of Ambulatory Blood Pressure with Mortality and Cardiovascular Events

Univariable linear Cox regression showed that both SBP and PP were risk factors for all endpoints of study participants, while DBP was not a risk factor for any endpoints (see online suppl. Table 1; for all online suppl. material, see www.karger.com/doi/10.1159/000510298). Multivariable Cox regression revealed that SBP was still an independent predictor of cardiovascular mortality and cardiovascular events but not for all-cause mortality after adjustments for age, sex, BMI, duration of PD, HbA1c, HOMA-IR, albumin, and NT-proBNP. Furthermore, DBP was still not a risk factor for any endpoints, while PP was still an independent predictor of all 3 endpoints after adjustments (Table 2).

The KM survival curves for all endpoints revealed a significant difference between the PP groups (Fig. 1A-C). Furthermore, univariable nonlinear Cox regression with restricted cubic spline showed the HRs for PP related to the median value (Fig. 2A-C). Similarly, we conducted the KM curves and restricted cubic spline for SBP (shown in online suppl. Fig. 1, 2A-C), which showed that the pattern for cardiovascular mortality and cardiovascular events is more obvious than that for all-cause mortality. As for DBP, there are no correlations between DBP and cardiovascular mortality and outcomes both in the KM curves and restricted cubic spline (shown in online suppl. Fig. 3, 4A-C). The above analysis confirmed the effects of PP and SBP on mortality and cardiovascular events. 


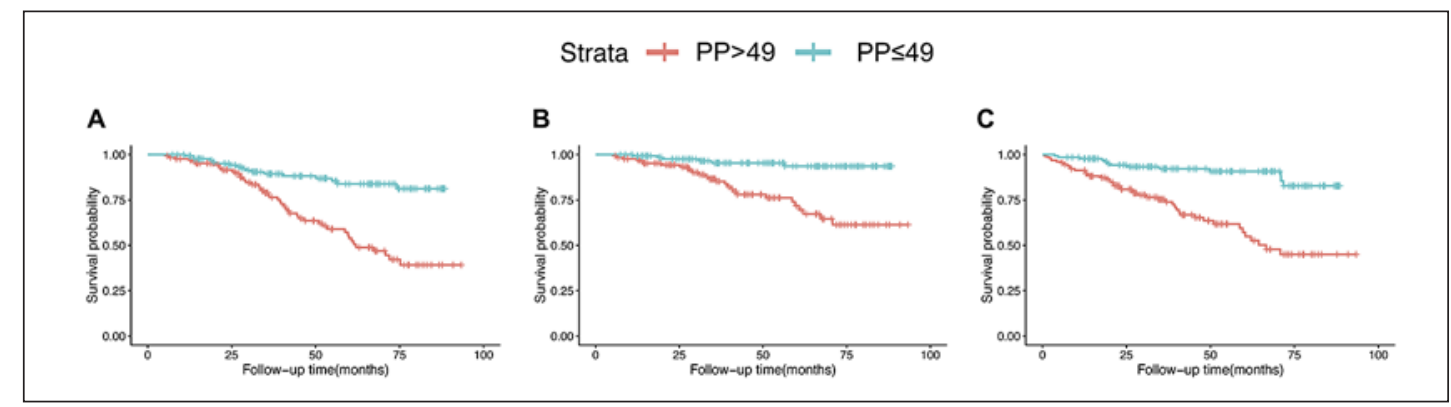

Fig. 1. Kaplan-Meier analysis of PP ( $\mathrm{mm} \mathrm{Hg})$ with all-cause mortality $(p<0.0001)$ (A), cardiovascular mortality $(p<0.0001)$ (B), and cardiovascular events $(p<0.0001)$ (C). The PD patients with higher PP levels were associated with higher all-cause mortality, cardiovascular mortality, and cardiovascular events. PP, pulse pressure; PD, peritoneal dialysis.

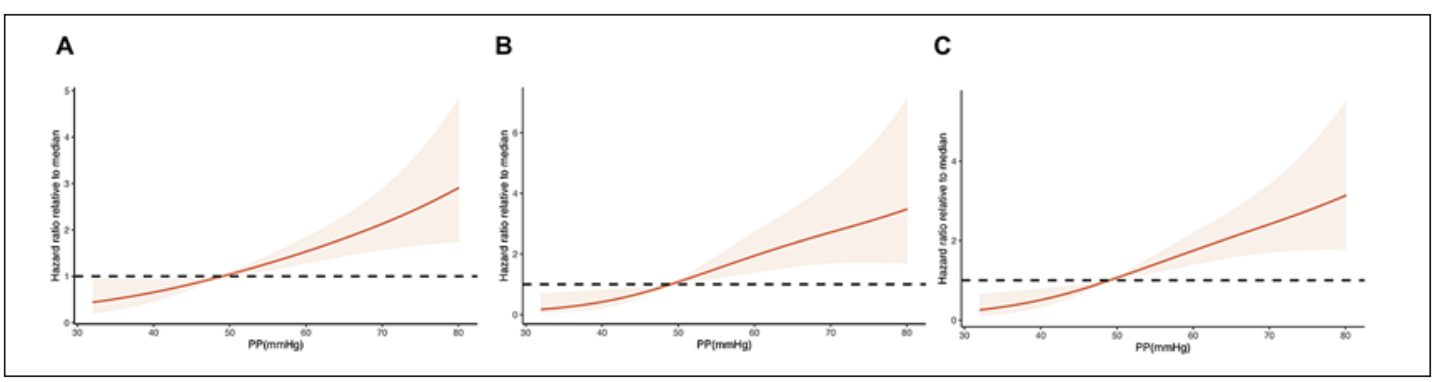

Fig. 2. Nonlinear Cox regression analysis using penalized smoothing splines for association of PP with allcause mortality (A), cardiovascular mortality (B), and cardiovascular events (C). The hazard ratio is represented by the solid line and the $95 \%$ CI by the shaded area. The PD patients with higher PP levels were associated with higher all-cause mortality, cardiovascular mortality, and cardiovascular events. PP, pulse pressure; PD, peritoneal dialysis.

Table 3. ORs for the likelihood of high pulse pressure in PD patients based on logistic regression analyses

\begin{tabular}{|c|c|c|c|c|}
\hline \multirow[t]{2}{*}{ Variable } & \multicolumn{2}{|l|}{ Univariable } & \multicolumn{2}{|l|}{ Multivariable } \\
\hline & OR $(95 \% \mathrm{CI})$ & $p$ value & OR $(95 \% \mathrm{CI})$ & $p$ value \\
\hline Age & $1.059(1.040-1.078)$ & $<0.001$ & $1.040(1.019-1.061)$ & $<0.001$ \\
\hline Sex & $1.197(0.729-1.963)$ & 0.477 & - & - \\
\hline Calcification & $5.898(3.452-10.077)$ & $<0.001$ & $3.069(1.632-5.772)$ & 0.001 \\
\hline NT-proBNP (per 1,000 pg/mL) & $1.082(1.033-1.132)$ & 0.001 & $1.074(1.026-1.124)$ & 0.002 \\
\hline HbA1c $(\%)$ & $2.042(1.495-2.789)$ & $<0.001$ & - & - \\
\hline HOMA-IR & $1.127(1.039-1.224)$ & 0.004 & - & - \\
\hline Serum albumin $(\mathrm{g} / \mathrm{dL})$ & $0.906(0.858-0.956)$ & $<0.001$ & - & - \\
\hline $\mathrm{LDL}(\mathrm{mmol} / \mathrm{L})$ & $1.410(1.027-1.936)$ & 0.034 & - & - \\
\hline Hemoglobin (g/dL) & $0.987(0.974-1.000)$ & 0.044 & - & - \\
\hline
\end{tabular}

OR, odds ratio; PD, peritoneal dialysis; NT-proBNP, N-terminal pro brain natriuretic peptide; HbA1c, glycated hemoglobin; HOMA-IR, homeostasis model of assessment-insulin resistance; LDL, low-density lipoprotein. 


\section{Risk Factors of Elevated PP}

According to the results in Table 1, age, sex, calcification, NT-proBNP, HbA1c, HOMA-IR, albumin, LDL, and hemoglobin were added to the model for multivariable logistic analysis. As listed in Table 3, we found that age, NT-proBNP, and the presence of calcification were independent factors associated with elevated PP.

\section{Discussion and Conclusion}

In the present study, the associations of ambulatory blood pressure recordings with mortality and cardiovascular events in patients on PD were evaluated. During the follow-up period, we identified that PP was an independent predictor of all-cause mortality, cardiovascular mortality, and cardiovascular events, and SBP was an independent predictor of cardiovascular mortality and cardiovascular events. Meanwhile, we investigated the risk factors associated with elevated PP, and it revealed that age, NT-proBNP, and the presence of calcification were significantly associated with elevated PP. These findings revealed that 24-h PP was a superior tool for the prediction of mortality and cardiovascular diseases compared with other blood pressure parameters in PD patients. To our knowledge, this is the largest prospective study conducted so far in the PD population, which revealed the associations of ambulatory blood pressure recordings with mortality and cardiovascular events in a long follow-up period.

Goldfarb-Rumyantzev et al. [14] found that low SBP and PP were associated with increased mortality in PD patients. We speculated that the reason for the discrepancy between our results and their findings was short follow-up times in their study (mean follow-up of 23 months). Some studies suggested that longer follow-up leads to a better understanding of the long-term effects of blood pressure on survival in dialysis patients, as hypertension was a significant risk factor for late mortality, which supports the findings in our current study [5, $6,15]$.

In our study, the association of SBP with all-cause mortality lost statistical significance after adjustment for NT-proBNP. NT-proBNP is not only a biomarker of left ventricular hypertrophy and ventricular dysfunction, but also able to mirror changes in fluid status [16, 17]. Fluid overload has long been known common among dialysis patients, which can also predict mortality independent of blood pressure $[18,19]$. Therefore, the strict volume control is vital for PD patients. Besides, the role of SBP in cardiovascular risks cannot be ignored in PD patients.

PP is generally considered a superior tool for the prediction of mortality and cardiovascular diseases compared with other blood pressure parameters in both general and dialysis populations [20,21]. Our study has reached similar conclusions, and possible explanation has been suggested: vascular calcification may have played an important role in the associations of PP with clinical outcomes in PD patients. Increased arterial stiffness is believed to be closely linked to elevated PP [22], and substantial evidence suggests the importance of vascular calcification in arterial stiffening [23-25]. In our previous study, coronary calcification was common in PD patients and coronary artery calcification score was an independent predictor of all-cause mortality, cardiovascular mortality, and cardiovascular events [26-28]. Hence, we believed that the tight association between vascular calcification and PP may potentially explain the associations of PP with all 3 study endpoints.

Several studies reported a U-shaped relationship between SBP or PP and mortality in patients on HD, while numerous cohort studies in the general population found a positive correlation between increased blood pressure and clinical outcomes [8-11]. Explanations for the U-shaped association in HD patients include that low blood pressure may be a marker for

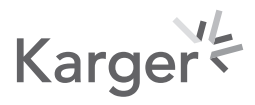


severe comorbidities including heart failure, atrial fibrillation, chronic inflammation, and malnutrition, which may have a major impact on outcomes [29]. In the current study, the U-shaped association between SBP or PP and mortality was not found, probably for the following reasons: patients in our study were in relatively good nutritional condition and the average albumin level of our study subjects is $36.2 \mathrm{~g} / \mathrm{L}$, which is significantly higher than that of other dialysis population. For example, a study by Balafa et al. [30] reported that the average albumin level of their PD patients was $33.9 \mathrm{~g} / \mathrm{L}$. Second, in contrast to hemodialysis patients, we have a lower prevalence of cardiac comorbidities including heart failure and atrial fibrillation. In the study about ambulatory blood pressure in HD patients conducted by Mayer et al. [11], the prevalence of heart failure and atrial fibrillation are 18 and 20\%, respectively. However, in our study, only $12(4.6 \%)$ participants had heart failure and $2(0.7 \%)$ had atrial fibrillation. Therefore, no hypotension occurred in our study subjects, leading to our results more similar to those of the general population.

Strengths of this study include the sample size, follow-up time, and inclusion of detection of calcification. However, certain limitations remained. First, our study was a single-center study and multicenter research is required to be conducted to confirm our conclusions. Second, the number of death and events was relatively small, which may affect the statistical power. Third, we did not analyze the effects of underlying cardiac diseases such as heart failure and atrial fibrillation on blood pressure due to too few events occurring during the period.

In conclusion, 24-h ambulatory PP was the most significant predictor of all blood pressure indicators for clinical outcomes in PD patients. Thus, we suggest that the goal of antihypertensive therapy in PD patients should be not only to decrease SBP but also to reduce PP. Furthermore, this study highlights the need to investigate measures that would lessen arterial stiffness and optimize volume control to improve clinical outcomes in PD patients.

\section{Acknowledgement}

The authors thank all patients for participating in this study.

\section{Statement of Ethics}

The study protocol was approved by the Ethics Committee of Huashan Hospital affiliated to Fudan University. All patients gave informed consent prior to the study.

\section{Conflict of Interest Statement}

The authors have no conflicts of interest to declare.

\section{Funding Sources}

This study was supported by National Natural Science Foundation of China (NSFC: 81670692 and 81700673 ). The funders had no role in the study design, data collection and analysis, the decision to publish, or the preparation of this paper. 


\section{Author Contributions}

S.D. collected, analyzed, and interpreted data, and drafted the manuscript. Y.C. interpreted the data and drafted the manuscript. D.S. and X.G. collected the data. C.H. and Q.X. coordinated and revised the manuscript. T.Z. conceived and designed the study, helped draft the manuscript, had full access to all the study data, and take responsibility for the integrity of the data and the accuracy of the analysis. All authors provided critical feedback and approved the final manuscript.

\section{References}

1 de Jager DJ, Grootendorst DC, Jager KJ, van Dijk PC, Tomas LM, Ansell D, et al. Cardiovascular and noncardiovascular mortality among patients starting dialysis. JAMA. 2009 Oct 28;302(16):1782-9.

2 Cocchi R, Degli Esposti E, Fabbri A, Lucatello A, Sturani A, Quarello F, et al. Prevalence of hypertension in patients on peritoneal dialysis: results of an Italian multicentre study. Nephrol Dial Transplant. 1999 Jun; 14(6):1536-40.

3 Krediet RT, Balafa O. Cardiovascular risk in the peritoneal dialysis patient. Nat Rev Nephrol. 2010 Aug;6(8): 451-60.

4 Agarwal R, Flynn J, Pogue V, Rahman M, Reisin E, Weir MR. Assessment and management of hypertension in patients on dialysis. J Am Soc Nephrol. 2014 Aug;25(8):1630-46.

5 Mazzuchi N, Carbonell E, Fernández-Cean J. Importance of blood pressure control in hemodialysis patient survival. Kidney Int. 2000 Nov; 58(5):2147-54

6 Udayaraj UP, Steenkamp R, Caskey FJ, Rogers C, Nitsch D, Ansell D, et al. Blood pressure and mortality risk on peritoneal dialysis. Am J Kidney Dis. 2009 Jan;53(1):70-8.

7 Parati G, Stergiou G, O’Brien E, Asmar R, Beilin L, Bilo G, et al. European Society of Hypertension practice guidelines for ambulatory blood pressure monitoring. J Hypertens. 2014 Jul;32(7):1359-66.

8 Banegas JR, Ruilope LM, de la Sierra A, Vinyoles E, Gorostidi M, de la Cruz JJ, et al. Relationship between clinic and ambulatory blood-pressure measurements and mortality. N Engl J Med. 2018 Apr 19;378(16):1509-20.

9 Yang WY, Melgarejo JD, Thijs L, Zhang ZY, Boggia J, Wei FF, et al. Association of office and ambulatory blood pressure with mortality and cardiovascular outcomes. JAMA. 2019 Aug 6;322(5):409-20.

10 Zager PG, Nikolic J, Brown RH, Campbell MA, Hunt WC, Peterson D, et al. "U” curve association of blood pressure and mortality in hemodialysis patients. Medical Directors of Dialysis Clinic, Inc. Kidney Int. 1998 Aug;54(2): 561-9.

11 Mayer CC, Matschkal J, Sarafidis PA, Hagmair S, Lorenz G, Angermann S, et al. Association of ambulatory blood pressure with all-cause and cardiovascular mortality in hemodialysis patients: effects of heart failure and atrial fibrillation. J Am Soc Nephrol. 2018 Sep;29(9):2409-17.

12 O'Brien E, Mee F, Atkins N. Evaluation of the Schiller BR-102 ambulatory blood pressure system according to the protocols of the British Hypertension Society and the Association for the Advancement of Medical Instrumentation. Blood Press Monit. 1999 Feb;4(1):35-43.

13 Denchev SV, Simova II, Matveev MG. Evaluation of the SCHILLER BR-102 plus noninvasive ambulatory blood pressure monitor according to the International Protocol introduced by the Working Group on Blood Pressure Monitoring of the European Society of Hypertension. Blood Press Monit. 2007 Oct;12(5):329-33.

14 Goldfarb-Rumyantzev AS, Baird BC, Leypoldt JK, Cheung AK. The association between BP and mortality in patients on chronic peritoneal dialysis. Nephrol Dial Transplant. 2005 Aug;20(8):1693-701.

15 Stidley CA, Hunt WC, Tentori F, Schmidt D, Rohrscheib M, Paine S, et al. Changing relationship of blood pressure with mortality over time among hemodialysis patients. J Am Soc Nephrol. 2006 Feb;17(2):513-20.

16 Kazanegra R, Cheng V, Garcia A, Krishnaswamy P, Gardetto N, Clopton P, et al. A rapid test for B-type natriuretic peptide correlates with falling wedge pressures in patients treated for decompensated heart failure: a pilot study. J Card Fail. 2001 Mar;7(1):21-9.

17 Wang AY, Lam CW, Yu CM, Wang M, Chan IH, Zhang Y, et al. N-terminal pro-brain natriuretic peptide: an independent risk predictor of cardiovascular congestion, mortality, and adverse cardiovascular outcomes in chronic peritoneal dialysis patients. J Am Soc Nephrol. 2007 Jan;18(1):321-30.

18 Ates K, Nergizoglu G, Keven K, Sen A, Kutlay S, Erturk S, et al. Effect of fluid and sodium removal on mortality in peritoneal dialysis patients. Kidney Int. 2001 Aug; 60(2): 767-76. https: //dx.doi. org/10.1046/j.1523-1755.2001.060002767.x.

19 Ozkahya M, Ok E, Toz H, Asci G, Duman S, Basci A, et al. Long-term survival rates in haemodialysis patients treated with strict volume control. Nephrol Dial Transplant. 2006 Dec;21(12):3506-13.

20 Klassen PS, Lowrie EG, Reddan DN, DeLong ER, Coladonato JA, Szczech LA, et al. Association between pulse pressure and mortality in patients undergoing maintenance hemodialysis. JAMA. 2002 Mar 27;287(12):154855 . 
21 Said MA, Eppinga RN, Lipsic E, Verweij N, van der Harst P. Relationship of arterial stiffness index and pulse pressure with cardiovascular disease and mortality. J Am Heart Assoc. 2018 Jan 22;7(2):e007621.

22 Mitchell GF, Conlin PR, Dunlap ME, Lacourcière Y, Arnold JM, Ogilvie RI, et al. Aortic diameter, wall stiffness, and wave reflection in systolic hypertension. Hypertension. 2008 Jan;51(1):105-11.

23 Guerin AP, London GM, Marchais SJ, Metivier F. Arterial stiffening and vascular calcifications in end-stage renal disease. Nephrol Dial Transplant. 2000 Jul;15(7):1014-21. https://dx.doi.org/10.1093/ndt/15.7.1014.

24 Toussaint ND, Lau KK, Strauss BJ, Polkinghorne KR, Kerr PG. Relationship between vascular calcification, arterial stiffness and bone mineral density in a cross-sectional study of prevalent Australian haemodialysis patients. Nephrology. 2009 Feb;14(1):105-12.

25 Jung CH, Lee WY, Kim SY, Jung JH, Rhee EJ, Park CY, et al. The relationship between coronary artery calcification score, plasma osteoprotegerin level and arterial stiffness in asymptomatic type 2 DM. Acta Diabetol. 2010 Dec;47 Suppl 1:145-52.

26 Shang D, Xie Q, Ge X, Yan H, Tian J, Kuang D, et al. Hyperphosphatemia as an independent risk factor for coronary artery calcification progression in peritoneal dialysis patients. BMC Nephrol. 2015 Jul 18;16:107.

27 Shang D, Xie Q, Shang B, Zhang M, You L, Hao CM, et al. Hyperphosphatemia and hs-CRP initiate the coronary artery calcification in peritoneal dialysis patients. Biomed Res Int. 2017;2017:2520510.

28 Xie Q, Ge X, Shang D, Li Y, Yan H, Tian J, et al. Coronary artery calcification score as a predictor of all-cause mortality and cardiovascular outcome in peritoneal dialysis patients. Perit Dial Int. 2016 Mar-Apr;36(2): 163-70.

29 Kalantar-Zadeh K, Ikizler TA, Block G, Avram MM, Kopple JD. Malnutrition-inflammation complex syndrome in dialysis patients: causes and consequences. Am J Kidney Dis. 2003 Nov;42(5):864-81.

30 Balafa O, Halbesma N, Struijk DG, Dekker FW, Krediet RT. Peritoneal albumin and protein losses do not predict outcome in peritoneal dialysis patients. Clin J Am Soc Nephrol. 2011 Mar;6(3):561-6. 